Copyright 2021 IEEE. Published in the IEEE 2021 International Conference on Image Processing (IEEE ICIP 2021), scheduled for 19-22 September 2021 in Anchorage, Alaska, United States. Personal use of this material is permitted. However, permission to reprint/republish this material for advertising or promotional purposes or for creating new collective works for resale or redistribution to servers or lists, or to reuse any copyrighted component of this work in other works, must be obtained from the IEEE. Contact: Manager, Copyrights and Permissions / IEEE Service Center / 445 Hoes Lane / P.O. Box 1331 / Piscataway, NJ 08855-1331, USA. Telephone: + Intl. 908-562-3966. DOI: 10.1109/ICIP42928.2021.9506766

\title{
WELSCH BASED MULTIVIEW DISPARITY ESTIMATION
}

\author{
James L. Gray, Aous T. Naman, David S. Taubman \\ School of Electrical Engineering and Telecommunications \\ Faculty of Engineering \\ University of New South Wales
}

\begin{abstract}
In this work, we explore disparity estimation from a high number of views. We experimentally identify occlusions as a key challenge for disparity estimation for applications with high numbers of views. In particular, occlusions can actually result in a degradation in accuracy as more views are added to a dataset. We propose the use of a Welsch loss function for the data term in a global variational framework for disparity estimation. We also propose a disciplined warping strategy and a progressive inclusion of views strategy that can reduce the need for coarse to fine strategies that discard high spatial frequency components from the early iterations. Experimental results demonstrate that the proposed approach produces superior and/or more robust estimates than other conventional variational approaches.
\end{abstract}

Index Terms - Disparity Estimation, Multiview, Welsch loss

\section{INTRODUCTION}

Disparity estimation has been studied extensively in the literature and many different approaches have been taken for this problem. Most existing approaches focus on stereo pairs and excellent results have been achieved in this context [1] [2] [3] [4]. However, the intuitive assumption that adding more views of a given scene will make disparity estimation more accurate does not actually hold. In scenes with occlusions, it turns out that as more views are added, with larger baselines, the increased areas of occlusion pose problems for most disparity estimation algorithms [5]. This is unfortunate, since larger baselines allow for greater precision in the estimated disparity [6].

Current hand-crafted methods of multiview disparity estimation with large numbers of views generally attempt to deal with this by using explicit occlusion based reasoning. This is done to reduce the influence of occluded areas on the estimated disparity. These techniques often use heuristics such as confidence based measures [7] or specific visibility reasoning [5] [8] [9]. These are often based on preliminary estimates of the disparity field themselves, so that early errors can potentially be reinforced. Other approaches treat occluded areas simply as outliers [10] [11] [12] and reduce their effect on the solution. This is conceptually similar to the use of robust cost functions in optical flow, reducing the influence of outliers on the solution without explicitly identifying them. Common robust cost functions include the Huber norm [13] and similar $L^{1}$-like norms [14] [15] the Geman and McClure norm, the Lorentzian norm [16], the Tukey norm [17] and the Welsch loss function [18].

In this paper we experimentally show that occlusions are a key problem for extending stereo disparity to a large number of views. We also propose a novel method of dealing with these occlusions in a multiview context using a Welsch loss function based data term. In particular, we propose an automatic selection process for Welsch loss parameter $\sigma_{d}$, a progressive approach to including the multiple views and a warping strategy that uses a disciplined multiple hypotheses method for upsampling the disparity field. We evaluate the proposed method using a synthetic dataset with 31 views of the same scene as well as a 4D Light Field Benchmark training dataset [19].

This paper is organised as follows. The proposed Welsch- $L^{1}$ multiview disparity algorithm is detailed in Section 2 The progressive inclusion of views is detailed in Section 3 . The warping strategy which uses a disciplined method for upsampling disparity fields is discussed in Section 4 The performance of the proposed algorithm is evaluated in Section 5 Conclusions are provided in Section6

\section{WELSCH- $L^{1}$ DISPARITY ESTIMATION}

For simplicity, we assume that we are dealing with images taken from cameras that are co-planar, with identical orientation. This means that the two dimensional disparity vector $\mathbf{d}_{p, q}(\mathbf{s})$, describing the apparent displacement between location $\mathbf{s}$ in view $p$ and a corresponding location in view $q$, can be written as

$$
\mathbf{d}_{p, q}(\mathbf{s})=\mathbf{B}_{p, q} \cdot F \cdot r(\mathbf{s})
$$

where $F$ is the focal length, $r(\mathbf{s})$ is the reciprocal depth or normalised disparity, and $\mathbf{B}_{p, q}$ is the baseline between views $p$ and $q$. It is more convenient to write this as

$$
\mathbf{d}_{p, q}(\mathbf{s})=\mathbf{B}_{p, q}^{\prime} \cdot w(\mathbf{s})
$$

where $\mathbf{B}^{\prime}$ and $w(\mathbf{s})$ are normalized baselines and reciprocal depth fields such that the nearest pair of views have $\mathbf{B}^{\prime} \in\{0,1\}^{2}$.

We apply a global variational approach, similar to [20] to minimise the energy of

$$
E=E_{d a t a}+\alpha^{2} E_{r e g},
$$

where $E_{\text {data }}$ is the data term, $E_{\text {reg }}$ is the regularisation term and $\alpha$ determines the relative significance of the regularisation term.

\subsection{Data term}

The use of the Welsch loss function has been shown previously to be highly effective in limiting the influence of outliers and occlusions on a flow field in the domain of optical flow [21]. In the domain of multiview disparity estimation with potentially large occluded areas, this turns out to be particularly valuable. We therefore define our data term as

$$
E_{\text {data }}=\int_{\Omega} \sum_{p} \phi_{\sigma_{d}}\left(I_{q}(\mathbf{s})-I_{p}\left(\mathbf{s}+\mathbf{B}_{p, q}^{\prime} w(\mathbf{s})\right)\right) d \mathbf{s},
$$

where $\Omega$ is the image domain, $q$ is set to be the single reference view, and $\phi_{\sigma_{d}}$ is the Welsch loss function with the parameter $\sigma_{d}$. This is defined as

$$
\phi_{\sigma_{d}}(x)=\sigma_{d}^{2}\left(1-\exp \left(-x^{2} / 2 \sigma_{d}^{2}\right)\right)
$$

For small $w(\mathbf{s})$, equation (4) can be linearised as

$$
E_{\text {data }}=\int_{\Omega} \sum_{p} \phi_{\sigma_{d}}\left(\left\langle\nabla I(\mathbf{s}), \mathbf{B}_{p, q}^{\prime}\right\rangle w(\mathbf{s})+\delta I_{p, q}(\mathbf{s})\right) d \mathbf{s},
$$


where $\left\langle\nabla I(\mathbf{s}), \mathbf{B}_{p, q}^{\prime}\right\rangle$ is the inner product of the gradient $\nabla I$ and the normalised baseline vector $\mathbf{B}_{p, q}^{\prime}$ and $\delta I_{p, q}(\mathbf{s})$ is the difference in image intensity between $I_{q}(\mathbf{s})$ and $I_{p}(\mathbf{s})$. For larger $w(\mathbf{s})$ values, a warping strategy is required and this is discussed in Section 4

\subsection{Regularisation term}

This paper primarily focuses on the data term, because the data term is what primarily changes when extra views are added. As shown in equation (7) below, we adopt the $L^{1}$ norm for our regularisation term, as a reasonable choice which does not introduce additional parameters that might complicate the analysis. Specifically

$$
E_{r e g}=\int_{\Omega} \psi(\|\nabla w(\mathbf{s})\|) d \mathbf{s}
$$

where $\psi(x)=x$.

\subsection{Optimisation}

To discuss our optimisation approach, we must introduce the numerical approximations we use in the energy function. To approximate a continuous $\nabla I$ we use Derivative of Gaussian filters in both the $x$ and $y$ directions $\left(G_{x, \sigma}, G_{y, \sigma}\right)$ with $\sigma=0.75$. We write this as

$$
\nabla I(\mathbf{s}) \approx\left[\left(G_{x, \sigma} *\left(I_{q}+I_{p}\right)\right)(\mathbf{s}),\left(G_{y, \sigma} *\left(I_{q}+I_{p}\right)\right)(\mathbf{s})\right] .
$$

This is equivalent to sampling the derivative of Gaussian blurred image, $G_{\sigma}(\mathbf{s}) *\left(I_{p}(\mathbf{s})+I_{q}(\mathbf{s})\right)$. Thus, we avoid aliasing associated with using discrete derivative operators.

Similarly, $\delta I_{p, q}$ is calculated by subtracting one image from the other and then blurring the image pair with a Gaussian filter $G_{\sigma}$,

$$
\delta I_{p, q}(\mathbf{s}) \approx G_{\sigma}(\mathbf{s}) *\left(I_{q}(\mathbf{s})-I_{p}(\mathbf{s})\right) .
$$

We use an Iteratively Reweighted Least Squares approach to minimise the Energy function. At each stage we use a conjugate gradient technique to solve the weighted Euler-Lagrange equation

$$
\begin{array}{r}
W_{d}(\mathbf{s})\left\langle\nabla I(\mathbf{s}), \mathbf{B}_{p, q}^{\prime}\right\rangle^{2} w(\mathbf{s})+W_{d}(\mathbf{s}) \delta I_{p, q}\left\langle\nabla I(\mathbf{s}), \mathbf{B}^{\prime}{ }_{p, q}\right\rangle \\
=W_{r}(\mathbf{s}) \alpha^{2} \nabla^{2} w(\mathbf{s}),
\end{array}
$$

where $W_{d}(\mathbf{s})$ and $W_{r}(\mathbf{s})$ are the data term and regularisation term weights and $\nabla^{2} w(\mathbf{s})$ is approximated using the discrete point spread function

$$
\left[\begin{array}{ccc}
1 / 12 & 1 / 6 & 1 / 12 \\
1 / 6 & -1 & 1 / 6 \\
1 / 12 & 1 / 6 & 1 / 12
\end{array}\right] .
$$

\section{PROGRESSIVE INCLUSION OF VIEWS}

Disparity is proportional to the baseline as per equation (1). If the outer views are considered initially, we may find that the disparity field $\mathbf{d}(\mathbf{s})$ is too large. This results in the linearisation in equation (6) becoming inaccurate as we will explain shortly. To prevent this, we propose an approach analogous to a coarse-to-fine strategy. We start with a subset of nearby views and then progressively include further away views, until all views are used. This allows the technique to include the important high resolution disparity information from the further away views, whilst preventing issues associated with large baselines from becoming a significant.

The linearisation in equation (6) is based on a Taylor series approximation of a disparity shift. To simplify matters we consider a constant normalised reciprocal depth $w_{0}$ with the shift performed on the reference view $I_{q}(\mathbf{s})$ so that,

$$
\left.I_{q}(\mathbf{s})-I_{q}\left(\mathbf{s}+\mathbf{B}_{p, q}^{\prime} w_{0}\right)\right) \approx\left\langle\nabla I_{q}(\mathbf{s}), \mathbf{B}_{p, q}^{\prime}\right\rangle w_{0} .
$$

We can take the Fourier transform of [12] as

$$
\hat{I}_{q}(\boldsymbol{\omega})-\hat{I}_{q}(\boldsymbol{\omega}) e^{j \boldsymbol{\omega}^{T} \mathbf{B}_{p, q}^{\prime} w_{0}} \approx \hat{I}_{q}(\boldsymbol{\omega}) j \boldsymbol{\omega}^{T} \mathbf{B}_{p, q}^{\prime} w_{0}
$$

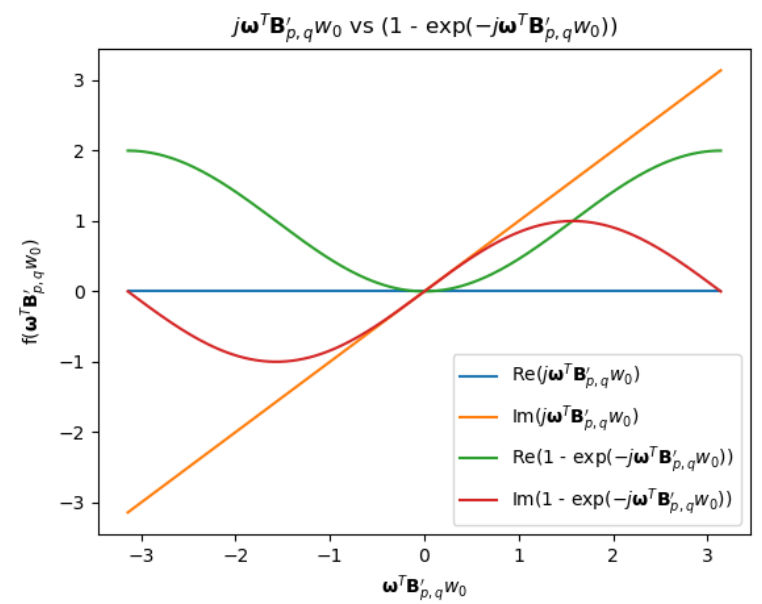

Fig. 1. $j \boldsymbol{\omega}^{T} \mathbf{B}_{p, q}^{\prime} w_{0}$ plotted alongside $\left(1-e^{-j \boldsymbol{\omega}^{T} \mathbf{B}_{p, q}^{\prime} w_{0}}\right)$.

where $\hat{I}_{q}$ is the Fourier transform of $I_{q}$ and he approximation in equation (13) relies on

$$
1-e^{j \boldsymbol{\omega}^{T} \mathbf{B}_{p, q}^{\prime} w_{0}} \approx j \boldsymbol{\omega}^{T} \mathbf{B}_{p, q}^{\prime} w_{0}
$$

As illustrated in Figure 1, when $\boldsymbol{\omega}^{T} \mathbf{B}_{p, q}^{\prime} w_{0}$ increases beyond $\pi / 2$ the approximation degrades.

One way of solving this problem is to use a coarse-to-fine strategy with spatial low-pass filtering [14] [22]. This ensures that $\hat{I}_{q}(\boldsymbol{\omega})$ is sufficiently bandlimited, effectively limiting $\boldsymbol{\omega}$. If done correctly, $\boldsymbol{\omega}^{T} \mathbf{B}_{p, q}^{\prime} w_{0}$ is kept small. Where required, such as where the disparities between adjacent views are too large, we apply this strategy.

Although spatial low-pass filtering is sometimes unavoidable, it has the strong disadvantage of discarding high frequency details that may be required to reduce the ambiguity of correspondences. For this reason, our proposed approach relies primarily on limiting $\mathbf{B}_{p, q}^{\prime}$, rather than $\omega$, by using views close to the reference view and then progressively increasing the number of views. At stage $s$, where $s \in 0,1,2, \ldots S$, we include a view $p$ if $\left\|\mathbf{B}_{p, q}^{\prime}\right\|_{\infty} \leq k+s c$, where $k$ and $c$ are constants. At each stage $s$, we perform IRLS optimisation to obtain an estimated disparity. We warp all views according to the estimated disparity, to ensure that at $s+1$, the error in the approximation in equation 13 is rather small.

It would be possible to extend this algorithm to use multiple different reference views when estimating the disparity. However, this would require fusing several depth estimates associated with different reference views and is therefore beyond the scope of this paper.

\section{WARPING STRATEGY}

To minimise the introduction of aliasing during warping, which is particularly problematic around strong discontinuities, we perform warping at twice the resolution of the images. We upsample the image using windowed-sinc interpolation. To similarly increase the resolution of the disparity we use a disciplined disparity upsampling approach.

The intuitive approach to upsampling a disparity field is to treat it the same as an image. Unlike image data, however, there is no reason to believe that disparity fields should exhibit bandlimited sampling properties. Instead of bandlimited interpolation, therefore, we propose a multi-hypothesis approach.

Specifically, we first use a nearest-neighbour policy to upsample the discrete disparity field by a factor of 2 and then hypothesize 9 high resolution disparity fields by applying unit shifts to the upsampled field. We can write this as

$$
w_{\mathbf{h}}[\mathbf{m}]=w_{\uparrow 2}[\mathbf{m}+\mathbf{h}],
$$


where $\mathbf{h} \in\{0, \pm 1\}^{2}, w_{\uparrow 2}$ is the initial upsampled disparity, and $w_{\mathbf{h}}$ is the candidate corresponding to shift $\mathbf{h}$.

We then warp the upsampled image, which is inherently smooth, using each of these disparity fields to produce 9 candidate images. These images are then averaged. This can be represented as

$$
I_{\uparrow, \mathcal{W}_{d}}[x, y]=\frac{1}{9} \sum_{h_{y}=-1}^{1} \sum_{h_{x}=-1}^{1} \mathcal{W}_{d_{\mathbf{h}}}\left(I_{\uparrow}\right)[x, y],
$$

where $d_{\mathbf{h}}=\mathbf{B}^{\prime} w_{\mathbf{h}}, I_{\uparrow}$ is the upsampled image and $\mathcal{W}_{d_{\mathbf{h}}}()$ is the bilinear warping operator. $I_{\uparrow, \mathcal{W}_{d}}$, is then decimated using windowedsinc filters to produce our final warped image.

\section{EVALUATION}

To fully implement our proposed algorithm, we select $\sigma_{d}$ by first estimating the squared error in the optical flow equation across the image domain for each view adjacent to the centre view. Specifically,

$$
\sigma_{d}=\frac{1}{\left|\mathcal{I}_{c}\right|} \sum_{p} \sqrt{\frac{1}{\left|\Omega_{p}\right|} \sum_{\Omega_{p}}\left(\left\langle\nabla I(\mathbf{s}), \mathbf{B}_{p, q}^{\prime}\right\rangle w^{(k)}(\mathbf{s})+\delta I_{p, q}\right)^{2}},
$$

where, $\mathcal{I}$ is the set of all views and $\mathcal{I}_{c}$ is the set of views adjacent to the centre view. We only use centre views because they are likely to have the smallest occluded areas. We calculate $\sigma_{d}$ each time we perform a reweighting step during IRLS optimisation.

We also impose a monotonicity constraint, explicitly preventing any increase in $\sigma_{d}$ as the iteration proceeds. This is reasonable, because we expect the solution for $w$ to become increasingly accurate with reweighting.

In order to realise $L^{1}$ cost functions in the IRLS optimisation framework, we actually employ a Huber function with linear transition point $\epsilon=0.0001$. This is used for the $L^{1}$ regularisation term, and when comparing our propose Welsh loss data term with $L^{1}$.

For comparison purposes, to ensure that the regularisation strength $\alpha$ has roughly the same weight across increasing numbers of views, we scale $\alpha$ by $\sqrt{|\mathcal{I}|}$.

We first use synthetic data to evaluate our algorithm using a dataset involving 31 views of the same scene. The spacing between views is $1.25 \mathrm{~mm}$ and they are 360 pixels tall and 640 pixels wide. The views are arranged along the $x$-axis. The focal length of the cameras is $50 \mathrm{~mm}$. We select the small spacing to avoid requiring a multi-resolution approach on this dataset. To simulate a real imaging process, we add Gaussian white noise of variance $\sigma_{n}^{2}=0.01$ to the images, where image intensity values are real and have the range $[0,1]$. The centre view of this dataset is shown in Figure 2 (a) and the Ground Truth disparity field is shown in Figure 2 (b). Note that the vertical bars produce obvious occlusions.

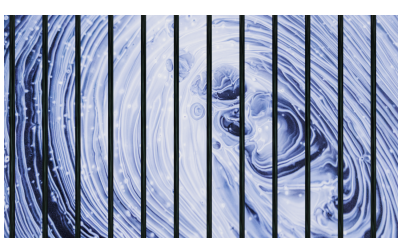

(a)

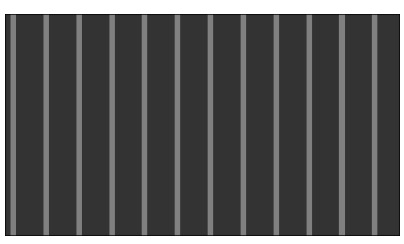

(b)
Fig. 2. The centre view in our dataset (a) and the corresponding Ground Truth disparity field (b).

The ground truth disparity field is twice the resolution of the estimated disparity field, so we use our disciplined method of upsampling disparity to evaluate our results, (see Section 4). We use the root mean square error, but we include the 9 different upsampled disparity hypotheses (indexed by $m$ )

$$
R M S E=\sqrt{\frac{1}{9|\Omega|} \sum_{m} \sum_{n}\left(r_{\text {est }}[n]-r_{g t}[n]\right)^{2}} .
$$

We denote our proposed model with a Welsch cost function for the data term and $L^{1}$ regularisation as Welsch- $L^{1}$. We compare it with an $L^{2}-L^{2}$ approach, an $L^{2}-L^{1}$ approach and an $L^{1}-L^{1}$ approach. The first norm refers to the cost function used for the data term, $\phi()$, and the second refers to the cost function used for the regularisation term, $\psi()$. For all three models, we use our progressive inclusion of views approach beginning with the 3 middle views and adding 2 more views at each stage. We chose the $\alpha$ values $0.01,0.025,0.05,0.1,0.2,0.5,1,2,5$ and ran each algorithm with each $\alpha$ value. The plots for a given approach in Figure 3 show the best error value for a given number of images over all $\alpha$ for that approach. This is necessary, because the optimum $\alpha$ value is generally different for each algorithm.

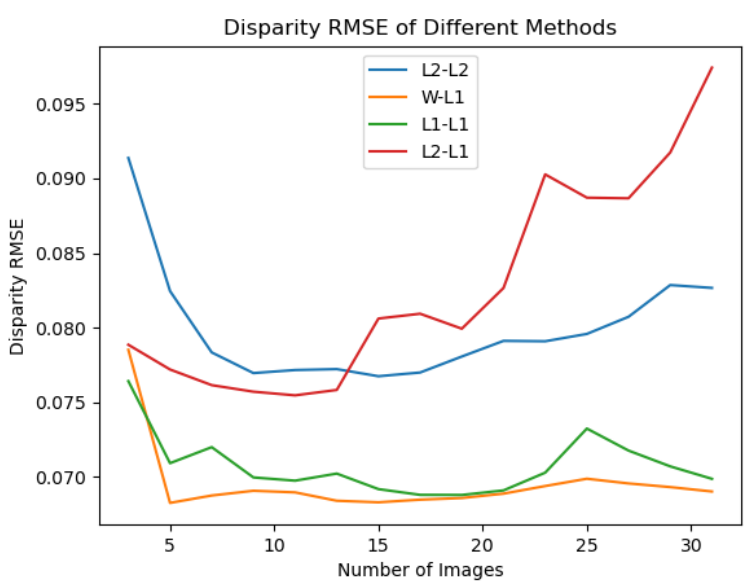

Fig. 3. RMSE results for the different approaches. $L^{2}$-based results have increasing RMSE values from 10 views onwards. For the Welsch $\left(W-L^{1}\right)$ and the $L^{1}-L^{1}$ approach, this does not occur. For large numbers of views the Welsch based method worked best.

Figure 3 shows that all $L^{2}$-based approaches have error levels that trend downward initially and then upward after roughly 10 views as more views are added. This is because in the initial stage, the noise in the images is uncorrelated so adding more images makes the data term more accurate. However, as we add views further and further apart we increase the size of occluded areas and with an $L^{2}$ data term this increasingly pollutes the disparity field. This is because occluded and non-occluded areas contribute with equal weight, pulling the solution towards their average.

With the $L^{1}-L^{1}$ norm, we see the initial downward trend, but no increase after roughly 10 views. We see similar, but improved results for the Welsch- $L^{1}$ approach compared to the $L^{1}-L^{1}$ results. We can see from Figure 4 (c) and (d) that the Welsch data term is better at determining where the background begins and ends when compared with the $L^{1}$ data term.

We also note that the $L^{1}-L^{1}$ lower bound involves results from four $\alpha$ values, while the Welsch- $L^{1}$ lower bound involves results from three. In Figure 5 we see that the $L^{1}-L^{1}$ results for a given $\alpha$ are less consistent than the Welsch- $L^{1}$ results. This is particularly important, since in real applications one has no way to know the best performing regularisation strength $\alpha$.

We also evaluate the performance of the proposed approach using a 4D Light Field Benchmark training dataset [19]. We compare the estimated disparity from our algorithm with the ground truth data 


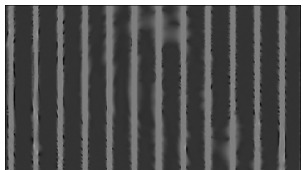

(a) $L^{2}-L^{2}$ disparity field

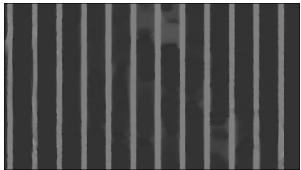

(c) $L^{1}-L^{1}$ disparity field

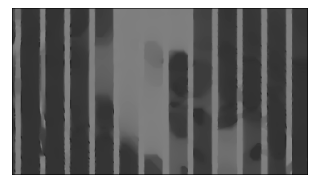

(b) $L^{2}-L^{1}$ disparity field

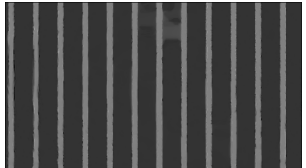

(d) Welsch- $L^{1}$ disparity field
Fig. 4. The best estimated disparity fields obtained with each algorithm on all 31 views.

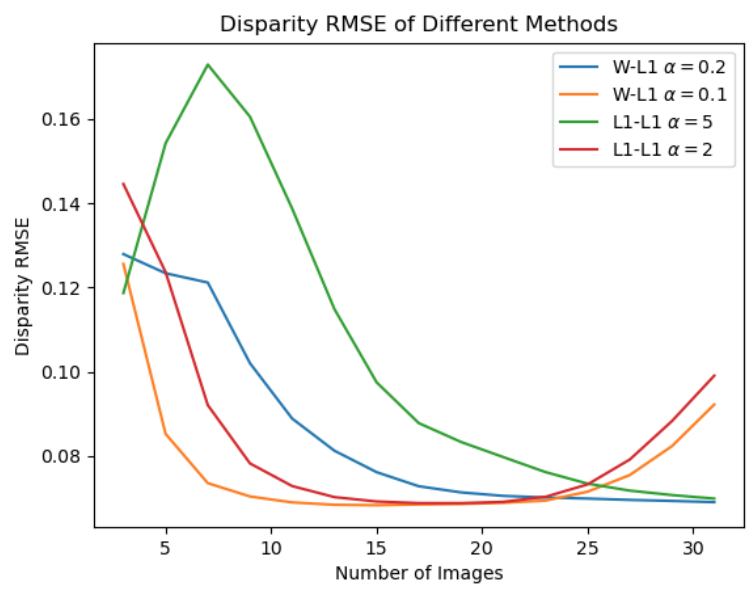

Fig. 5. RMSE results for the Welsch- $L^{1}$ and $L^{1}-L^{1}$ for a few values for $\alpha$. Note that the $L^{1}-L^{1}$ algorithm provides good results for a smaller number of views than the Welsch- $L^{1}$ algorithm. At present, we do not have an explanation for the worsening of the $L^{1}-L^{1}$ results for $\alpha=5$ up to around 7 to 9 views.

which has a resolution that is the same as that of the image. We use $\alpha=0.01,0.025,0.05,0.1,0.2,0.5,1,2,5$ for all four approaches.

Because the ground truth field is the same resolution as the images, we simply use RMSE to evaluate the performance of our algorithm. Our progressive inclusion of views strategy begins with selecting the central view and a neighbouring view. We then add views one a time, vertically and horizontally only, leading to a crosshair camera array. At each stage we produce a disparity estimate. In total, we use 17 of the 81 views. In Table 1 we detail the RMSE results for our four algorithms on each of the training scenes and we show the disparity fields in Figure 6

\begin{tabular}{|c|cccc|}
\hline Scene & $L^{2}-L^{2}$ & $L^{2}-L^{1}$ & $L^{1}-L^{1}$ & Welsch- $L^{1}$ \\
\hline Boxes & 0.349 & 0.446 & $\mathbf{0 . 3 0 9}$ & 0.317 \\
\hline Cotton & 0.249 & 0.315 & 0.209 & $\mathbf{0 . 1 7 7}$ \\
\hline Dino & 0.117 & 0.130 & $\mathbf{0 . 1 0 9}$ & 0.117 \\
\hline Sideboard & 0.223 & 0.336 & $\mathbf{0 . 2 0 6}$ & 0.221 \\
\hline Average & 0.234 & 0.307 & $\mathbf{0 . 2 0 8}$ & $\mathbf{0 . 2 0 8}$ \\
\hline
\end{tabular}

Table 1. RMSE values for 17 views for each scene with the best $\alpha$ value selected over all of the scenes.

With 17 views, the $L^{1}-L^{1}$ and the Welsch- $L^{1}$ algorithms have the same average RMSE (see Table 1] which is better than the other methods. The key advantage of the Welsch- $L^{1}$ algorithm in this dataset is that it is more consistent over a greater range of views for a given $\alpha$ value than the other algorithms tested. It has the best

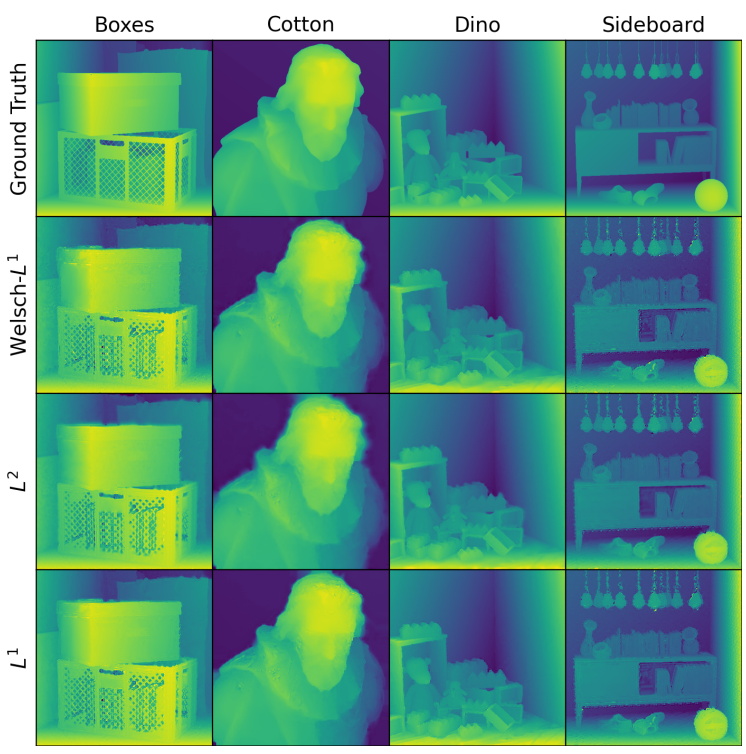

Fig. 6. Ground truth and estimated disparity fields with 17 views of four scenes from the 4D Light Field Training Dataset [19]. The colour scale is shared between the plots of the same scene, but not between scenes.

\begin{tabular}{|c|ccccc|}
\hline Method & Stereo & 5 views & 9 views & 13 views & 17 views \\
\hline$L^{2}-L^{2}$ & 0.307 & 0.245 & 0.228 & 0.228 & 0.234 \\
\hline$L^{2}-L^{1}$ & 0.530 & 0.384 & 0.314 & 0.285 & 0.307 \\
\hline$L^{1}-L^{1}$ & 0.397 & 0.238 & 0.214 & 0.207 & $\mathbf{0 . 2 0 8}$ \\
\hline Welsch- $L^{1}$ & $\mathbf{0 . 2 4 9}$ & $\mathbf{0 . 2 1 0}$ & $\mathbf{0 . 2 0 0}$ & $\mathbf{0 . 2 0 3}$ & $\mathbf{0 . 2 0 8}$ \\
\hline
\end{tabular}

Table 2. RMSE values averaged over all four scenes for different numbers of views (including the reference view). We choose the $\alpha$ value that gives the best 17 view RMSE value on average.

RMSE for all numbers of views shown in Table 2, reaffirming the results we find in our synthetic dataset.

This observed lack of consistency in the $L^{1}$ results may be caused by the $L^{1}$ estimator's breakdown point of 50\% [23]. With large numbers of views some areas may be occluded in around $50 \%$ of view pairs. Therefore the $L^{1}$ estimator will be almost at the breakdown point before considering any noise or other non-idealities in these areas. Additionally, with small numbers of views, the areas may be occluded in a greater proportion of view pairs, further contributing to the inconsistency in the $L^{1}-L^{1}$ results. The Welsch loss function does not have a breakdown point because infinite distances are not possible in a Welsch metric space [24] and this better limits the influence of these problematic areas on disparity estimation.

\section{CONCLUSION}

In this paper we experimentally show that occlusions present a significant problem for multiview disparity estimation algorithms. These occlusions can cause some algorithms, such as those that use an $L^{2}$ norm for the data-term to perform worse when more views are used. We propose a Welsch- $L^{1}$ disparity estimation algorithm as a possible solution to this problem. We also define a disciplined warping strategy and method for progressively including views which can be used to avoid removing high frequency content from views. Even in cases with very high numbers of views we show that these approaches produce excellent results both on our synthetic dataset and a 4D Light Field Dataset [19] when compared with the $L^{2}-L^{2}$, $L^{2}-L^{1}$ and $L^{1}-L^{1}$ approaches. 


\section{REFERENCES}

[1] D. Scharstein, R. Szeliski, and R. Zabih, "A taxonomy and evaluation of dense two-frame stereo correspondence algorithms," Proceedings - IEEE Workshop on Stereo and MultiBaseline Vision, SMBV 2001, , no. 1, pp. 131-140, 2001.

[2] Gengshan Yang, Joshua Manela, Michael Happold, and Deva Ramanan, "Hierarchical deep stereo matching on highresolution images," in Proceedings of the IEEE Conference on Computer Vision and Pattern Recognition, 2019, pp. 55155524.

[3] Xiao Song, Xu Zhao, Liangji Fang, Hanwen Hu, and Yizhou Yu, "Edgestereo: An effective multi-task learning network for stereo matching and edge detection," International Journal of Computer Vision, pp. 1-21, 2020.

[4] Huaiyuan Xu, Xiaodong Chen, Haitao Liang, Siyu Ren, Yi Wang, and Huaiyu Cai, "CrossPatch-Based Rolling Label Expansion for Dense Stereo Matching," IEEE Access, vol. 8, pp. 63470-63481, 2020.

[5] Sing Bing Kang, Richard Szeliski, and Jinxiang Chai, "Handling occlusions in dense multi-view stereo," Proceedings of the IEEE Computer Society Conference on Computer Vision and Pattern Recognition, vol. 1, pp. 103-110, 2001.

[6] Masatoshi Okutomi and Takeo Kanade, "A multiple-baseline stereo," IEEE Transactions on pattern analysis and machine intelligence, vol. 15, no. 4, pp. 353-363, 1993.

[7] C. Strecha and L. Van Gool, "Pde-based multi-view depth estimation," in Proceedings. First International Symposium on 3 D Data Processing Visualization and Transmission, 2002, pp. 416-425.

[8] Zhaokun Zhu, Christos Stamatopoulos, and Clive S. Fraser, "Accurate and occlusion-robust multi-view stereo," ISPRS Journal of Photogrammetry and Remote Sensing, vol. 109, pp. 47-61, nov 2015.

[9] Xiaoyan $\mathrm{Hu}$ and Philippos Mordohai, "Least commitment, viewpoint-based, multi-view stereo," Proceedings - 2nd Joint 3DIM/3DPVT Conference: 3D Imaging, Modeling, Processing, Visualization and Transmission, 3DIMPVT 2012, pp. 531538, 2012.

[10] Christian Bailer, Manuel Finckh, and Hendrik P.A. Lensch, "Scale robust multi view stereo," Lecture Notes in Computer Science (including subseries Lecture Notes in Artificial Intelligence and Lecture Notes in Bioinformatics), vol. 7574 LNCS, no. PART 3, pp. 398-411, 2012.

[11] Neill D F Campbell, George Vogiatzis, Carlos Hernández, and Roberto Cipolla, "Using Multiple Hypotheses to Improve Depth-Maps for Multi-View Stereo," in Computer Vision ECCV 2008, David Forsyth, Philip Torr, and Andrew Zisserman, Eds., Berlin, Heidelberg, 2008, pp. 766-779, Springer Berlin Heidelberg.

[12] Hae Gon Jeon, Jaesik Park, Gyeongmin Choe, Jinsun Park, Yunsu Bok, Yu Wing Tai, and In So Kweon, "Accurate depth map estimation from a lenslet light field camera," Proceedings of the IEEE Computer Society Conference on Computer Vision and Pattern Recognition, vol. 07-12-June, pp. 1547-1555, 2015.

[13] David Shulman and Jean-Yves Herve, "Regularization of discontinuous flow fields," in Proc. workshop on visual motion, 1989, pp. 81-86.
[14] Thomas Brox, Andrés Bruhn, Nils Papenberg, and Joachim Weickert, "High accuracy optical flow estimation based on a theory for warping," in European conference on computer vision. Springer, 2004, pp. 25-36.

[15] Sebastian Volz, Andres Bruhn, Levi Valgaerts, and Henning Zimmer, "Modeling temporal coherence for optical flow," in 2011 International Conference on Computer Vision. IEEE, 2011, pp. 1116-1123.

[16] Michael J. Black and Anand Rangarajan, "On the unification of line processes, outlier rejection, and robust statistics with applications in early vision," International Journal of Computer Vision, vol. 19, no. 1, pp. 57-91, 1996.

[17] Jean-Marc Odobez and Patrick Bouthemy, "Robust multiresolution estimation of parametric motion models," Journal of visual communication and image representation, vol. 6 , no. 4, pp. 348-365, 1995.

[18] Paul W Holland and Roy E Welsch, "Robust regression using iteratively reweighted least-squares," Communications in Statistics - Theory and Methods, vol. 6, no. 9, pp. 813-827, 1977.

[19] Katrin Honauer, Ole Johannsen, Daniel Kondermann, and Bastian Goldluecke, "A dataset and evaluation methodology for depth estimation on 4D light fields," in Asian Conference on Computer Vision 2016, 2016, vol. 10113 LNCS, pp. 19-34.

[20] Berthold K.P. P Horn and Brian G. Schunck, "Determining optical flow," Artificial Intelligence, vol. 281, pp. 319-331, 1981.

[21] Sean I. Young, Aous T. Naman, and David Taubman, "Graph Laplacian Regularization for Robust Optical Flow Estimation," IEEE Transactions on Image Processing, vol. 29, pp. 39703983, 2019.

[22] Enric Meinhardt-Llopis, Javier Sánchez Pérez, and Daniel Kondermann, "Horn-Schunck Optical Flow with a Multi-Scale Strategy," Image Processing On Line, vol. 3, pp. 151-172, 2013.

[23] Hendrik P Lopuhaa, Peter J Rousseeuw, et al., "Breakdown points of affine equivariant estimators of multivariate location and covariance matrices," The Annals of Statistics, vol. 19, no. 1, pp. 229-248, 1991.

[24] P L Davies and U Gather, "The Breakdown Point — Examples and Counterexamples," REVSTAT Statistical Journal, vol. 5, no. 1, pp. 1-17, 2007. 